Article

\title{
Insight into the Effect of TDMs on the Tribological Behaviors of the Ionic Liquid Composite Films
}

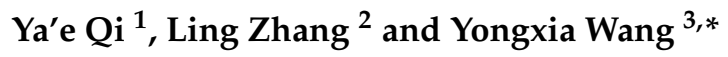 \\ 1 College of Chemistry and Chemical Engineering, Hexi University, Key Laboratory of Hexi Corridor \\ Resources Utilization of Gansu, Zhangye 734000, China; qiyaezhu@163.com \\ 2 Shenzhen CONE Tech. Co., Ltd. 51\&52 Building, Software Town of Shenzhen Universiade Longgong, \\ Shenzhen 518100, China; zhangling@chinacone.com \\ 3 College of Environmental Science and Engineering, Donghua University, 2999 Ren'min North Road, \\ Shanghai 201620, China \\ * Correspondence: wyx912@dhu.edu.cn
}

Received: 5 December 2019; Accepted: 30 December 2019; Published: 2 January 2020

\begin{abstract}
Ionic liquid (IL) combined with 2D materials has evoked considerable attention in the field of lubrication applications because of their speical structure and outstanding lubrication properties. However, the ambiguous effect of the 2D materials on the friction and anti-wear properties of the IL needs futher study. Here, we have obtained two families of IL composite films with additives of $\mathrm{MoS}_{2}$ and graphene via a combined process of spin-coated and curing, and the distinction of the effects of two additives on the tribological performance of the IL films was studied. The friction tests showed that the friction coefficient and anti-wear life of the IL films were greatly enhanced after the addition of $\mathrm{MoS}_{2}$ or graphene, which could be attributed to the improved load-carrying capacity and the second lubrication phase. Under a low addition content, graphene had more advantages to reduce the friction of the films, and $\mathrm{MoS}_{2}$ was more beneficial to the tribological properties with the additional content increased. The films with low friction and good anti-wear properties may be valuable for the rational design of lubrication films for the practical engineering applications.
\end{abstract}

Keywords: ionic liquid film; $\mathrm{MoS}_{2}$ nanosheet; graphene; friction; anti-wear

\section{Introduction}

Industrial equipment and devices are in constant motion, but the severe wear and interfacial adhesion have become great challenges for the moving and contact parts in the mechanical moving equipment [1-3]. Good lubrication has become a crucial issue for improving performance reliability and reducing the energy consumption of the moving parts, and a solid lubricant on the contact surface of moving parts has become an important choice. Since the initial report in 2001 [4], ionic liquids (IL) with special configuration and outstanding tribological performance have evoked a great deal of attention in the area of lubricating applications [5-10].

Based on the current reports, it has been supposed that the IL film could be used as a kind of important lubricant to reduce the friction and improve the anti-wear for the moving parts $[6,11,12]$. However, under the boundary lubricated condition, the insufficient load-carrying capacity and anti-wear performance of the IL films greatly limited their wide applications [13]. For the past few years, a lot of additives have been reported to enhance the tribological performance of IL with low friction coefficient and improved anti-wear ability [14-17]. Two-dimensional materials (TDMs), such as graphene and molybdenum disulfide $\left(\mathrm{MoS}_{2}\right)$, with outstanding anti-wear and ultralow friction coefficient, have become attractive solid lubricants to be used as additives to enhance a lubricating property of mechanical components [18-21]. 
The microtribological results of the lubricating composite film based on IL and graphene demonstrate that the friction coefficient and anti-wear ability of the IL film decrease with the graphene content increased, and the enhanced tribological performance could be ascribed to the synergic effect of IL and graphene in the composite films [22]. IL composite films with the functionalized graphene which could be evenly dispersed in IL without overlapping exhibited an improved load-carrying capacity and anti-wear compared with the pure IL film, attributing to the synergic effect between the additives and IL acted as the load-carrying and lubricating phase, respectively [14]. Moreover, it has been suggested that the composite film of IL/functionalized-graphene expands the application scope of IL lubrication film and shows an important application value in the moving parts [23]. In our previous work, it has been found that the $\mathrm{MoS}_{2}$ nanosheets as additives could greatly enhance the anti-wear properties of the IL films, attributing to the synergistic effect of the IL and $\mathrm{MoS}_{2}$ [24].

Combining the present reports, the appropriate amount of TDMs (graphene and $\mathrm{MoS}_{2}$ ) as additives show a great potential to enhance the tribological performance of IL film, and the corresponding mechanism of additives affecting the reduced friction and improving anti-wear properties is mainly ascribed to the improved load-carrying capacity of the composite films. Comparing the lubrication effect of graphene and $\mathrm{MoS}_{2}$, it has been considered that $\mathrm{MoS}_{2}$ nanosheets possessed an advantage over graphene, as the latter performed relatively higher and less stable tribological properties [25]. However, there are few works about the difference of two kinds of additives (graphene and $\mathrm{MoS}_{2}$ ) on the tribological properties of the IL-based films. Meanwhile, although the tribological performance and lubrication mechanism of the IL films have already been thoroughly described in other literature, the effect of lubricating additives on friction and anti-wear properties of IL based films still needs to be studied further.

Herein, with the aim to study the effect of 2D materials (TDMs) as additives on the tribological properties of the IL composite films, two kinds of nanosheets (graphene and $\mathrm{MoS}_{2}$ ) have been added in the IL composite films. The friction and anti-wear properties of series of the IL films with a different added amount of TDMs were studied, and the effect of the two lubricating additives on tribological properties of IL films were analyzed. The comparison of the effect of graphene and $\mathrm{MoS}_{2}$ acting as additives on the tribological performance of the IL films may be valuable for the rational design of IL-based lubricants in the practical lubrication applications.

\section{Materials and Methods}

\subsection{Preparation of the IL Composite Films}

The liquid exfoliation process of the $\mathrm{MoS}_{2}$ nanosheets has been described in the previous work [24]. Typically, a certain amount of the bulk $\mathrm{MoS}_{2}(100 \mathrm{mg}, 15 \mu \mathrm{m}$, Nanjing MKNANO Tech. Co., Ltd, Nanjing, China) and DMF ( $\mathrm{C}_{3} \mathrm{H}_{7} \mathrm{NO}, 30 \mathrm{~mL}$, Aladdin Reagents Co., Ltd, Shanghai, China) were exposed to ultrasound treatment for $4 \mathrm{~h}$, and then the obtained $\mathrm{MoS}_{2}$ suspensions were centrifuged at a speed of $2000 \mathrm{rpm}$ for $20 \mathrm{~min}$. The upper centrifuged suspension was extracted under 12,000 rpm for $30 \mathrm{~min}$, and, finally, a few-layer $\mathrm{MoS}_{2}$ nanosheets were cleared with ethanol and deionized water 3 times, respectively.

The formation of the IL ([BMIM] $\mathrm{BF}_{4}$, Sinopharm Chemical Reagent Co., Ltd, Ningbo, China) composite films added with $\mathrm{MoS}_{2}$ and graphene (Nanjing XFNANO Tech. Co., Ltd, Nanjing, China) was shown in Figure 1. The typical process was listed as follows: a different amount of obtained $\mathrm{MoS}_{2}$ nanosheets and graphene were dispersed into the isopropyl alcohol solution of IL ( $5 \mathrm{mg} / \mathrm{mL}$ ) under continuous ultrasonication, and the ultimate mass ratios of $\mathrm{MoS}_{2}$ or graphene in IL were $0.1 \mathrm{wt} \%, 0.3 \mathrm{wt} \%, 0.5 \mathrm{wt} \%, 1 \mathrm{wt} \%$, and $2 \mathrm{wt} \%$, respectively. The IL composite films were coated on the hydroxylated $\mathrm{Si} / \mathrm{SiO}_{2}$ wafers (obtained by immersing into the freshly prepared piranha solution $3\left(98 \% \mathrm{H}_{2} \mathrm{SO}_{4}\right) / 1\left(30 \% \mathrm{H}_{2} \mathrm{O}_{2}\right)$ at $90{ }^{\circ} \mathrm{C}$ for $30 \mathrm{~min}$.) by means of spin coating, and were heated at $120{ }^{\circ} \mathrm{C}$ for $30 \mathrm{~min}$ in $\mathrm{N}_{2}$. Finally, the obtained composite films of IL with $\mathrm{MoS}_{2}$ were named IL/0.1M, 
IL/0.3M, IL/0.5M, IL/1M, IL/2M, respectively, and the films of IL with graphene were IL/0.1G, IL/0.3G, IL/0.5G, IL/1G, IL/2G, respectively.

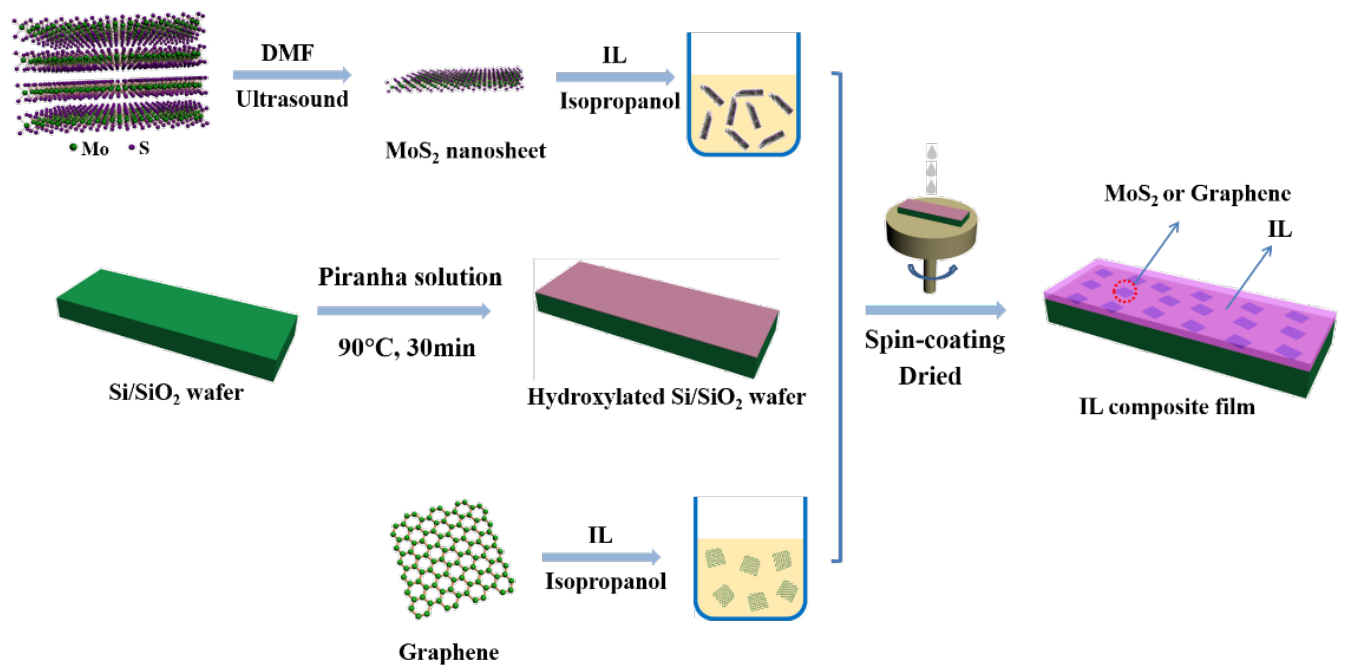

Figure 1. Preparation of the IL/MoS 2 or IL/graphene composite films via a spin-coating process.

\subsection{Characterization}

A Transmission Electron Microscope (TEM, JEM-2100F) was used to observe the morphology of the $\mathrm{MoS}_{2}$ and graphene. The thickness and morphology of the $\mathrm{MoS}_{2}$ nanosheets and graphene were obtained via Atomic Force Mcroscope (AFM, Asylum Research MFP-3D). The microstructure of the $\mathrm{MoS}_{2}$ nanosheets and graphene was studied by Raman spectra (inVia-Reflex, excitation wavelength: $532 \mathrm{~nm})$.

\subsection{Tribological Tests}

The tribological tests of a series of IL composite films were carried out with a ball-disk friction tester (HSR-2M). Under the frequency of $4.2 \mathrm{~Hz}$ and reciprocating distance of $5 \mathrm{~mm}$, the steel balls (GCr15) with a diameter of $6 \mathrm{~mm}$ were used to run against the IL composite films with a load of $100 \mathrm{~g}$. Each pair was tested thrice to take the average value. Optical microscopy was used to observe the worn scar of the steel balls and composite films, and the information of the chemical component on the worn scar of the ball sliding with the IL/0.3M and IL/0.3G was determined via XPS (escalab 250xi, Thermo Fisher Scientific).

\section{Results and Discussion}

The morphology of the $\mathrm{MoS}_{2}$ and graphene are observed via TEM, and the typical images are shown in Figure 2a,b. From the TEM images, it can be seen that both $\mathrm{MoS}_{2}$ and graphene have a nanosheet structure. AFM is applied to confirm the thickness of exfoliated $\mathrm{MoS}_{2}$ nanosheets and graphene, and the typical Raman spectra of the $\mathrm{MoS}_{2}$ nanosheets and graphene are shown in Figure S1.

For $\mathrm{MoS}_{2}$ shown in Figure 2c, it has a step height of individual layers of $3.5 \mathrm{~nm}$, which is compatible with the $0.62 \mathrm{~nm}$ for a single layer of the $\mathrm{MoS}_{2}$ crystal [26], and thus the layer number of the exfoliated $\mathrm{MoS}_{2}$ nanosheets is about 5. The above results show that the $\mathrm{MoS}_{2}$ produced by exfoliated process has a few-layers nanosheet structure, which is consistent with the previous reports for exfoliated $\mathrm{MoS}_{2}$. From the AFM image of the graphene shown in Figure 2d, it can be seen that a layer structure of graphene is formed. The cross-sectional height reveals that the thickness of the graphene is $1.1 \mathrm{~nm}$, and, according to the previous reported thickness of $0.34 \mathrm{~nm}$ [27], the layer number is about 3 .

The average size of the $\mathrm{MoS}_{2}$ and graphene are shown in Figure 3, from which it can be seen that the size of the $\mathrm{MoS}_{2}$ nanosheets is about $220 \mathrm{~nm}$. The average size of graphene is $395 \mathrm{~nm}$, which is 
much higher than that of the $\mathrm{MoS}_{2}$. The results show that the exfoliated $\mathrm{MoS}_{2}$ nanosheets have a relatively small particle size.
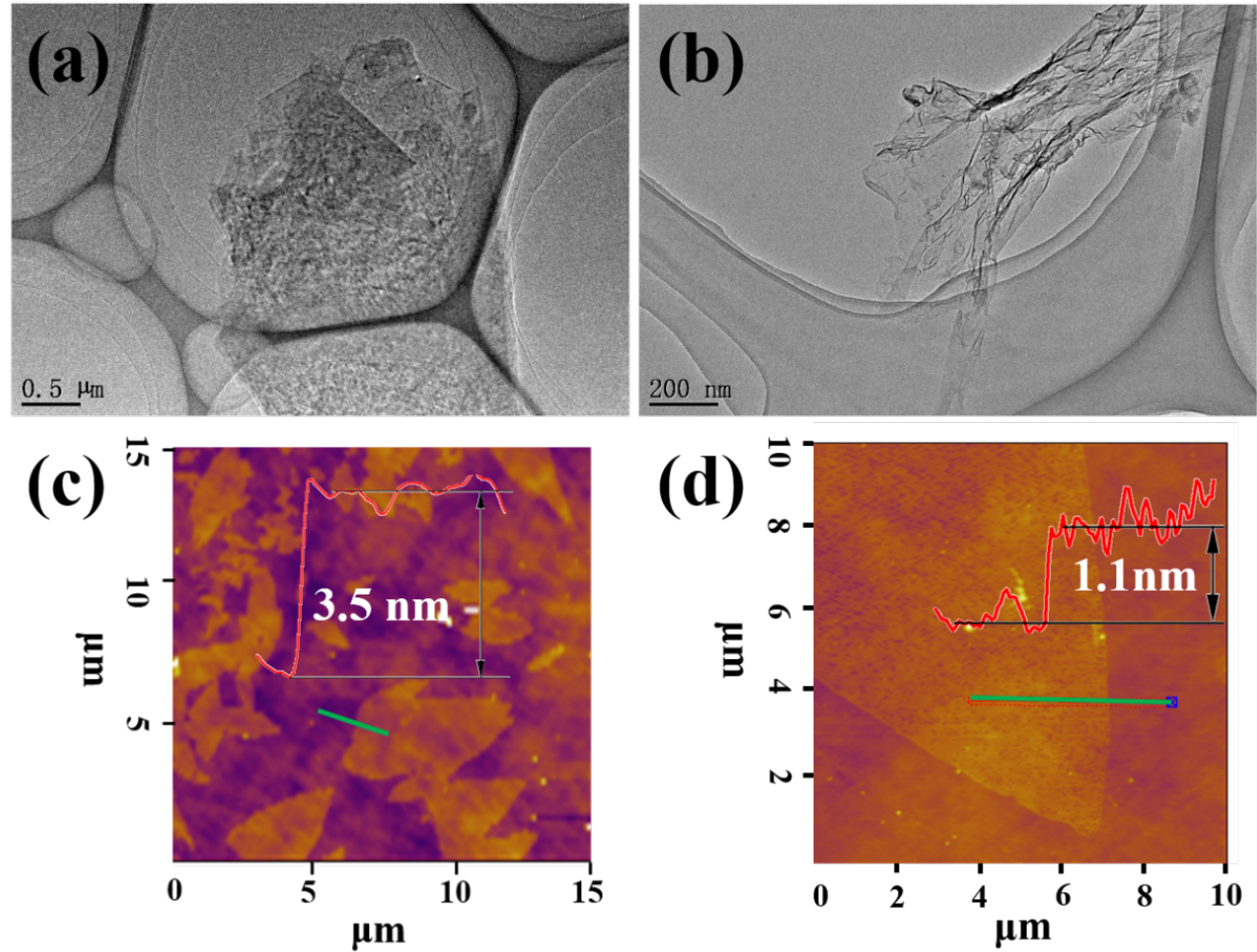

Figure 2. TEM and AFM images of the $\operatorname{MoS}_{2}(\mathbf{a}, \mathbf{c})$ and graphene $(\mathbf{b}, \mathbf{d})$.
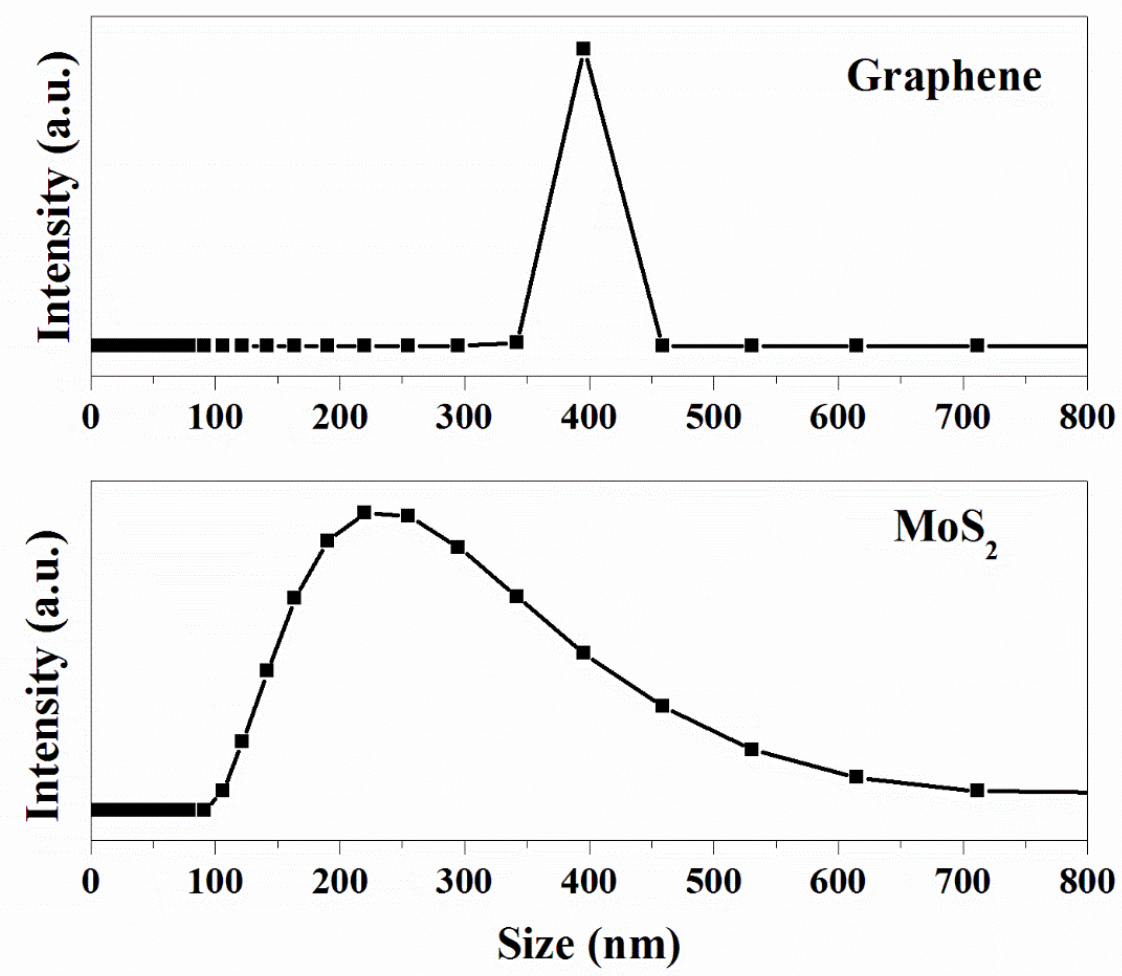

Figure 3. Average size distribution of the $\mathrm{MoS}_{2}$ nanosheets and graphene. 
The friction and anti-wear properties for the series of the IL composite films with $\mathrm{MoS}_{2}$ and graphene are tested by a friction tester under the load of $100 \mathrm{~g}$. Figure $4 \mathrm{a}, \mathrm{b}$ give the relation between the friction coefficient with sliding time of the IL composite films with $\mathrm{MoS}_{2}$ and graphene, respectively, from which, it can be seen that the pure IL film exhibits a relatively high friction coefficient up to 0.1 , and, more importantly, it shows an extreme short anti-wear life, ascribing to the poor load-carrying capacity. For the composite films with the addition of $\mathrm{MoS}_{2}$, they show improved anti-wear life and reduced friction coefficient. Combining the results reported in the previous work, the IL composite film with $\mathrm{MoS}_{2}$ content increased to $0.3 \mathrm{wt} \%$, and the anti-wear life of the composite film of IL/0.3M increases 10 times. The friction coefficient of the composite film increases gradually with the additional content of $\mathrm{MoS}_{2}$ increased, and, for the composite film with $2 \mathrm{wt} \% \mathrm{MoS}_{2}$, the anti-wear life dramatically reduces.
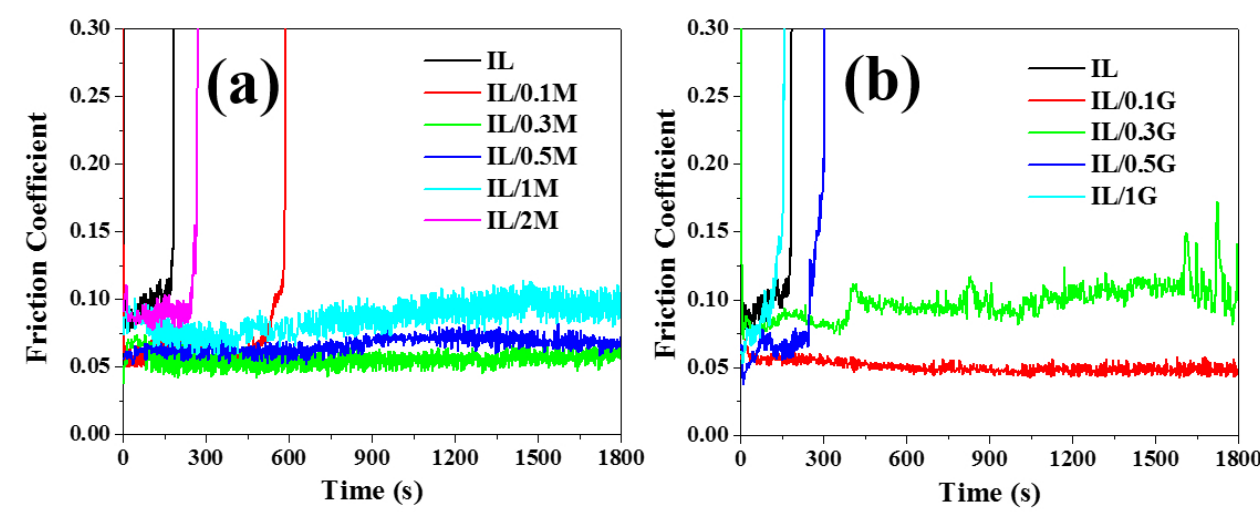

Figure 4. Friction coefficient as a function of sliding time for the IL films with different amounts of $\mathrm{MoS}_{2}$ nanosheets (a) and graphene (b).

The results of the friction test for the IL composite films with a different additional content of graphene are shown in Figure $4 b$, and it can be seen that a relatively low content of graphene $(0.1 \mathrm{w} t \%)$ can greatly reduce the friction coefficient while prolonging the anti-wear life. However, with the content of graphene increased to $0.3 \mathrm{wt} \%$, the friction coefficient of the composite films increases, and the anti-wear life of the composite films with $0.5 \mathrm{wt} \%$ exhibits a sharp decrease.

Based on the above results, appropriate content $\mathrm{MoS}_{2}$ nanosheets and graphene can greatly enhance the friction and anti-wear properties of the IL films. For the IL composite films with a low content of the additives $(0.1 \mathrm{wt} \%)$, graphene has more advantages for reducing the friction coefficient and improving the anti-wear life. With the increase of the content of the additives $(0.3 \mathrm{wt} \%), \mathrm{MoS}_{2}$ is more beneficial to the tribological properties.

The surface morphology of the worn scar for the series of the IL composite films with $\mathrm{MoS}_{2}$ are given in Figure 5, and it can be observed that the IL/0.3M film shows the smallest wear width. With the increase of additional content of the $\mathrm{MoS}_{2}$, the wear width increases gradually. For the steel ball sliding with the composite films, the wear scar diameter exhibits a similarity with the trend of wear width of the composite films. The IL composite film with high content of $\mathrm{MoS}_{2}$ shows poor wear resistance, indicating that the $\mathrm{MoS}_{2}$ may play a negative impact on the tribological performance of the IL films.

The worn morphology of the composite films of IL with different content of graphene is observed via optical microscope and shown in Figure 6. It can be seen that the wear scar of IL/0.1G is much smaller than that of the composite film of IL/0.3G. Compared to the results of the wear diameter of the steel ball sliding with IL composite films with $0.3 \mathrm{wt} \% \mathrm{MoS}_{2}$ and graphene, the IL/0.3M shows a smaller value than that of IL/0.3G, indicating that, under the same content of the additives, $\mathrm{MoS}_{2}$ has more advantages for improving the anti-wear ability of the films. Meanwhile, it can be easily observed 
that the worn scar of the steel ball is coated by a layer of transferred materials, and, at the same time, a large amount of wear debris gathered on the side of the wear scar of the IL/0.3G.

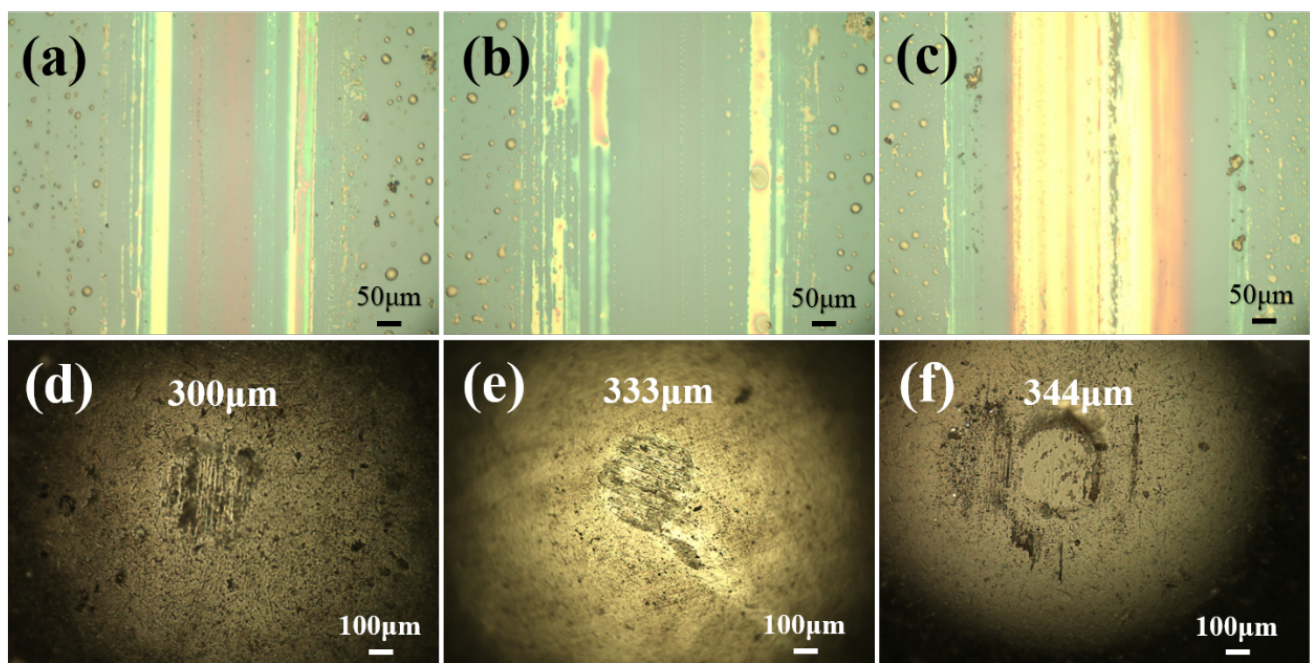

Figure 5. The worn morphology of the IL/M composite films and steel balls: IL/0.3M (a,d), IL/0.5M $(\mathbf{b}, \mathbf{e})$, and IL/1M (c,f).
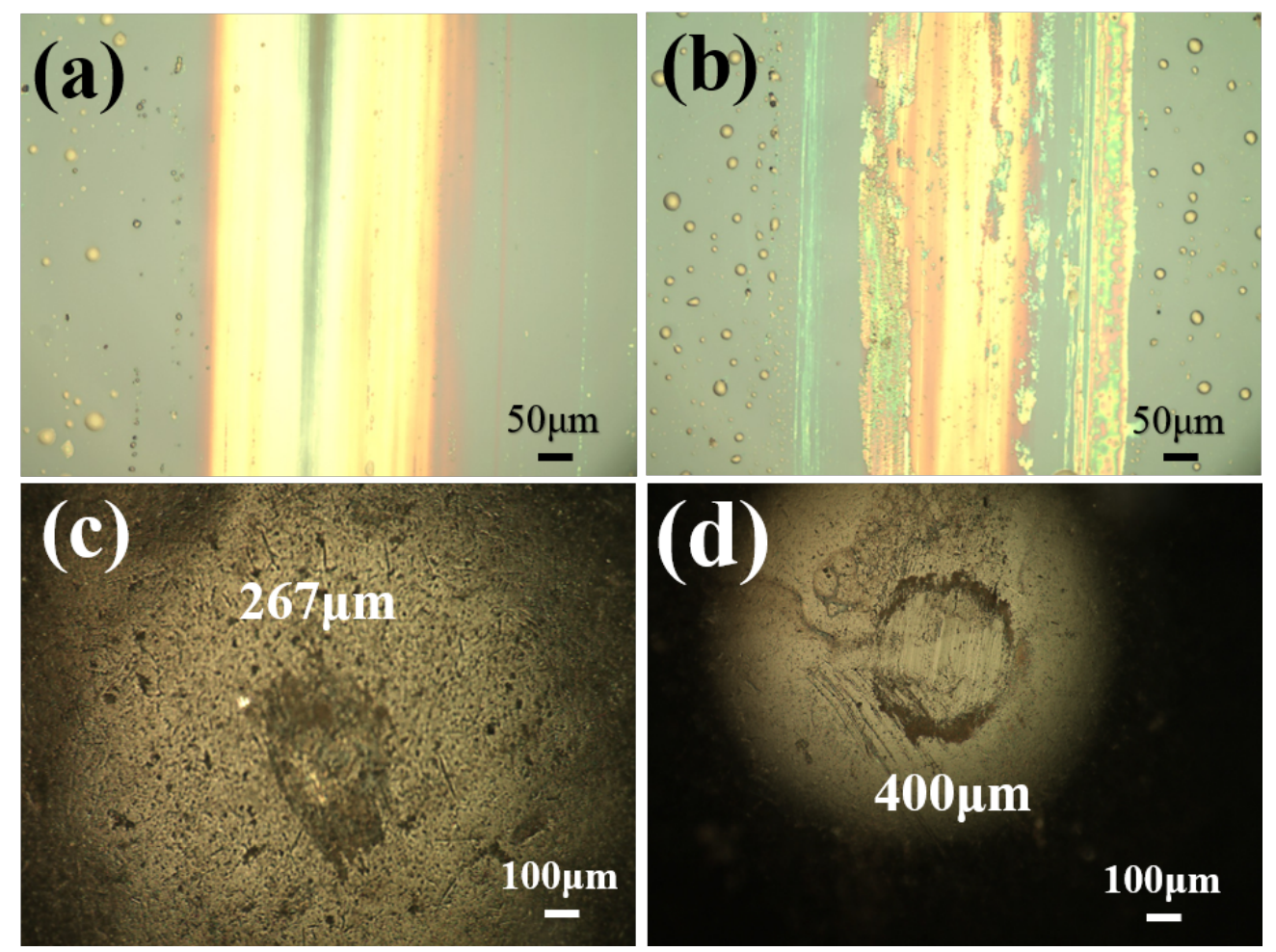

Figure 6. The morphology of the worn scar for the IL/G composite films and corresponding steel balls: IL/0.1G (a,c) and IL/0.3G (b,d).

In most cases, wear scar diameter (WSD) has been used to estimate to wear behaviors of the lubricants, and, from the WSD, the wear volume $(V)$ can be determined by the relations as follows [28,29] by:

$$
V=\left(\frac{\pi h}{6}\right)\left(\frac{3 d^{2}}{4}+h^{2}\right)
$$




$$
h=r-\operatorname{sqrt}\left(r^{2}-\frac{d^{2}}{4}\right),
$$

where $d$ and $r$ are the wear scar diameter and the radius of the steel ball, respectively.

Based on the above equations and the measured wear scar diameter $(d)$ of the steel ball, $V$ is calculated and the corresponding values are listed in Table 1. For the IL composite with $\mathrm{MoS}_{2}$, the IL/0.3M composite film shows the smallest wear volume, and the wear volume of the IL/M gradually becomes large with the increase of the content of $\mathrm{MoS}_{2}$, indicating that the high addition content of $\mathrm{MoS}_{2}$ exhibits a adverse effect on the tribological performance of the IL films. For the IL film with $0.1 \mathrm{wt} \%$ graphene $(\mathrm{IL} / 0.1 \mathrm{G})$, it shows a relatively small value of $V$. With the increase of graphene content to $0.3 \mathrm{wt} \%$, the wear volume of the composite film greatly increases, and it shows a five-fold larger than that of the IL with $0.1 \mathrm{wt} \%$.

Table 1. Wear volume $(V)$ of the steel ball sliding with different IL composite films.

\begin{tabular}{cccccc}
\hline & IL/0.3M & IL/0.5M & IL/1M & IL/0.1G & IL/0.3G \\
\hline$r(\mathrm{~mm})$ & 3 & 3 & 3 & 3 & 3 \\
$d(\mathrm{~mm})$ & 0.3 & 0.333 & 0.344 & 0.267 & 0.4 \\
$h(\mathrm{um})$ & 375 & 462 & 493 & 297 & 667 \\
$V\left(10^{-6} \mathrm{~mm}^{3}\right)$ & 133 & 201 & 229 & 83 & 419 \\
\hline
\end{tabular}

The chemical component on the worn scar of the steel balls sliding with the IL composite films is studied via XPS, in order to explore the lubrication mechanism of the 2D nanosheets as additives for the IL films. From the survey spectrum of the two composite films shown in Figure 7a, it can be seen that two obvious peaks existed at $399.8 \mathrm{eV}$ and $194 \mathrm{eV}$, corresponding to N1s and B1s peaks, can be attributed to the $\mathrm{N}$ and $\mathrm{B}$ in $\mathrm{IL}\left(\mathrm{C}_{8} \mathrm{H}_{15} \mathrm{~N}_{2} \mathrm{BF}_{4}\right)$. The above result indicates that the element of the IL could be transferred to the contact surface of the steel ball during the sliding with IL composite films. Additionally, the Mo3d and S2p peaks at $232.3 \mathrm{eV}$ and $161.8 \mathrm{eV}$ can be detected in the wear scar, indicating that the products of Mo and $\mathrm{S}$ in the IL/0.3M films transferred to the steel ball.

The detailed information of the $\mathrm{C} 1$ peaks are given in Figure $7 \mathrm{~b}$, and the Gaussian method is used to fit the corresponding peaks. The main peak centered at $284.5 \mathrm{eV}$ is assigned to $\mathrm{C}$ in graphene, indicating that graphene would be transferred to the wear scar of the steel ball. In the meantime, the weak signal of $\mathrm{C}-\mathrm{O}$ and $\mathrm{O}-\mathrm{C}=\mathrm{O}$ peaks at $286.4 \mathrm{eV}$ and $288.6 \mathrm{eV}$, respectively, is detected, which may be attributed to the oxidation of the graphene during sliding, or the oxycarbides on the steel ball exposed to air. For the Mo 3d, two peaks at $232.8 \mathrm{eV}$ and $228.8 \mathrm{eV}$ are assigned to Mo $3 \mathrm{~d} 3 / 2$ and $3 \mathrm{~d} 5 / 2$ of $\mathrm{MoS}_{2}$, and a weak signal of $\mathrm{MoO}_{3}$ at $234 \mathrm{eV}$ is detected, indicating that the transferred $\mathrm{MoS}_{2}$ is oxidized lightly. For the S2p, S 2p1/2 and 2p3/2 can be seen at $163.8 \mathrm{eV}$ and $161.3 \mathrm{eV}$, attributing to $\mathrm{S}$ in $\mathrm{MoS}_{2}$. From the above results, it can be concluded that transferred IL and $\mathrm{MoS}_{2}$ or graphene exist on the worn surface of the steel balls sliding with the IL/0.3M or IL/0.3G.

Based on the results of the tribological tests and the characterization of the worn surface of the steel balls sliding with series of the IL composite films, the greatly enhanced friction and anti-wear properties of the IL/M films can be attributed to the improved load-carrying capacity and the formed transfer films on the worn scar of the steel ball (Figure 8). Under a given load, the worse tribological properties of pure IL film may be ascribed to the poor load-carrying capacity of the films. For the IL composite films with 2D nanosheets as additives, they can greatly improve the load-carrying capacity of the composite film and thus give it longer anti-wear life than the IL film. Additionally, $\mathrm{MoS}_{2}$ or graphene as an effective solid lubricant may also act as the second lubricating phase to further enhance the friction and anti-wear properties of the composite films. The improved load-carrying capacity and second lubricating phase give the composite films a low friction coefficient and prolonged anti-wear life. 

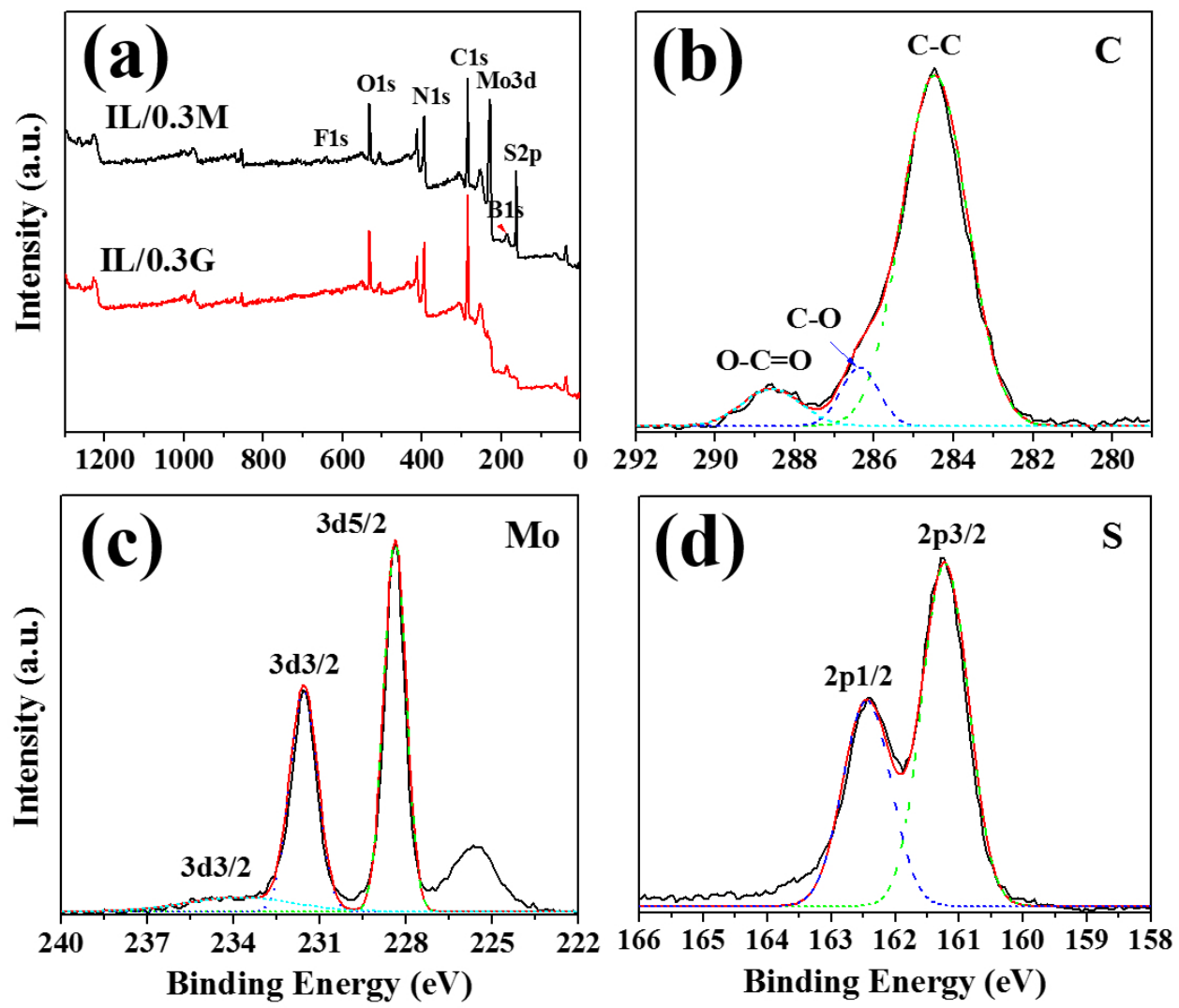

Figure 7. XPS survey spectra (a) of the wear scar of the steel balls sliding with IL/0.3M and IL/0.3G, C1s for ball-IL/0.3G (b), and Mo3d (c), S2p (d) for ball-IL/0.3M.

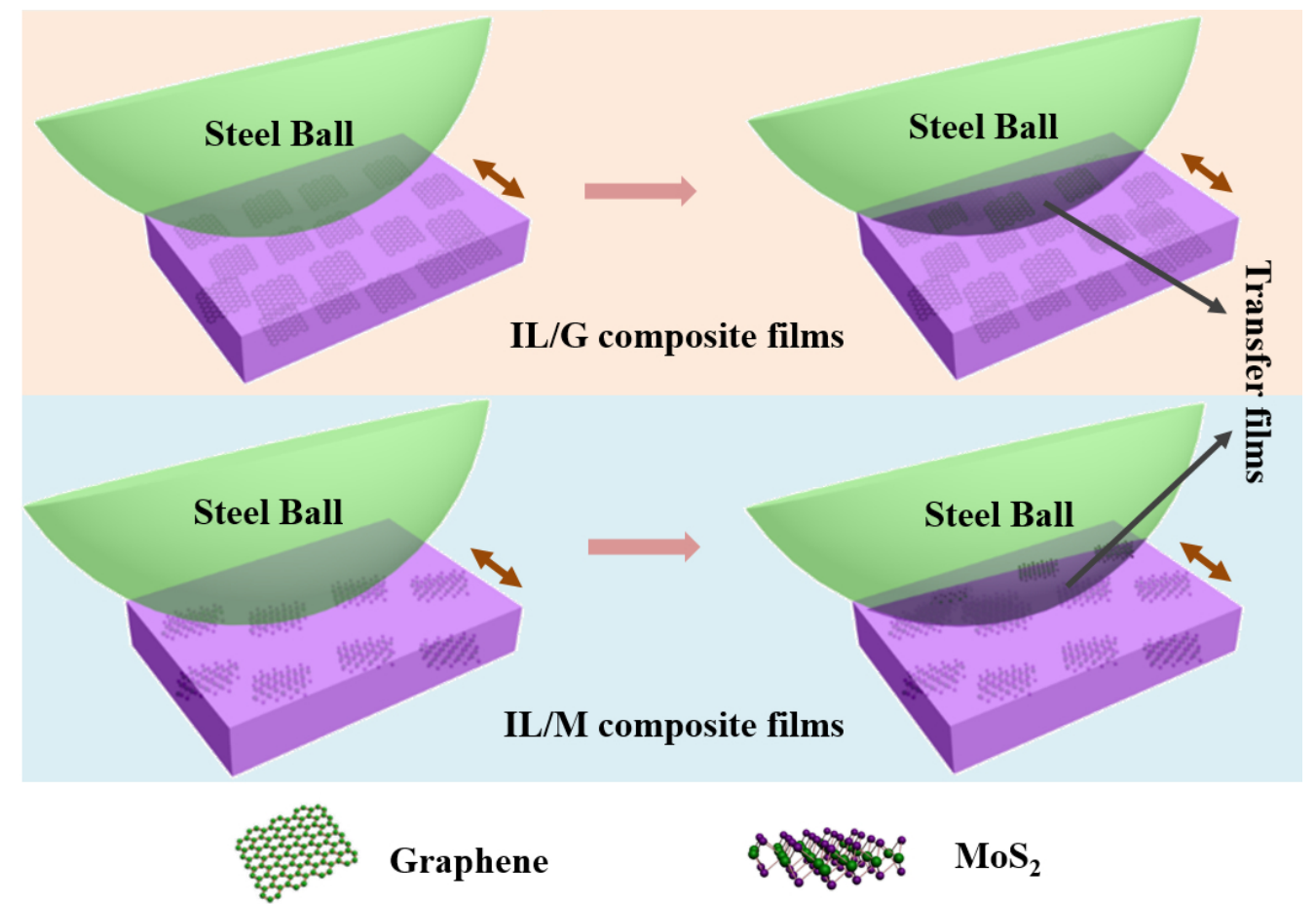

Figure 8. Schematic illustration of the tribological mechanism of the IL composite films. 
For the IL composite films with a low content of additives, graphene has more advantages to improve the tribological properties of the films, and $\mathrm{MoS}_{2}$ is more beneficial to the tribological properties with the increase of the addition content. Graphene is one-atom thick carbon arranged in a honeycomb structure, and $\mathrm{MoS}_{2}$ has a trilayer structure with one Mo atomic layer sandwiched between two $S$ atomic layers [30,31]. Under the same content of the additives, specific surface area of graphene is much higher than that of the $\mathrm{MoS}_{2}$ nanosheets, and thus a greater amount (volume) of graphene exists in the same volume of IL composite films. The addition of nanosheet in the IL composite films plays a disadvantageous effect on the film continuity, and excess graphene has a negative impact on the structure and continuity of the IL composite films. The morphology of the IL composite films with a different amount of $\mathrm{MoS}_{2}$ or graphene is shown in Figure S2, from which it can be seen that the surface morphology of the IL film becomes rough with the content of graphene increased to $1 \mathrm{wt} \%$. The deterioration of the film continuity would give a poor load-carrying capacity and eventually lead to the degradation of the tribological performance of the IL composite films (as shown in Figure S3).

\section{Conclusions}

A series of IL based composite films composed of IL and TDMs ( $\mathrm{MoS}_{2}$ or graphene) were obtained and the tribological properties of the composite films were studied. The friction coefficient and anti-wear life of the IL films were greatly improved with $\mathrm{MoS}_{2}$ or graphene as additives, and the enhanced tribological properties of the composite films were ascribed to the improved load-carrying capacity and the second lubrication phase. Graphene has more advantages for reducing friction of the films with a low content of additives, and, with the increase of the additional content, $\mathrm{MoS}_{2}$ is more beneficial to the tribological properties of the composite films. Excess addition of the additives would cause the deterioration of the film continuity and a poor load-carrying capacity, and eventually lead to the degradation of the tribological performance of the IL composite films.

Supplementary Materials: The following are available online at http:/ /www.mdpi.com/1996-1944/13/1/191/s1, Figure S1. Raman spectra of the $\mathrm{MoS}_{2}$ nanosheets and graphene; Figure S2. Morphology of the IL composite films observed by optical microscope: (a) IL, (b) IL/0.1M, (a) IL/1M, (a) IL/0.1G, (a) IL/1G; Figure S3. The wear process of the nonuniformity IL composite with excess additives.

Author Contributions: Conceptualization, Y.Q. and Y.W.; methodology, Y.W.; formal analysis, Y.Q. and L.Z.; data curation, Y.W.; writing-original draft preparation, Y.Q. and Y.W.; writing-review and editing, Y.Q. and Y.W. All authors have read and agreed to the published version of the manuscript.

Funding: This research was funded by the Science and Technology Planning Project of Gansu Province (18JR3RG203), the Peacock Team Project of Shenzhen Overseas High-Level Talents Innovation and Entrepreneurship Fund (KQTD2016112917131100), and Fundamental Research Funds for the Central Universities (2232018D3-10).

Conflicts of Interest: The authors declare no conflict of interest.

\section{References}

1. Izabela, S.; Michael, C.; Robert, W.C. Recent advances in single-asperity nanotribology. J. Phys. D Appl. Phys. 2008, 41, 123001.

2. Bhushan, B.; Israelachvili, J.N.; Landman, U. Nanotribology: Friction, wear and lubrication at the atomic scale. Nature 1995, 374, 607-616.

3. Bhushan, B. Nanotribology and nanomechanics of MEMS/NEMS and bioMEMS/BioNEMS materials and devices. In Nanotribology and Nanomechanics: An Introduction; Springer: Cham, Switzerland, 2017; pp. $797-907$.

4. Ye, C.; Liu. W.; Chen, Y.; Yu, L. Room-temperature ionic liquids: A novel versatile lubricant. Chem. Commun. 2001, 21, 2244-2245. [CrossRef]

5. Bonhote, P.; Dias, A.P.; Papageorgiou, N.; Kalyanasundaram, K.; Grätzel, M. Hydrophobic, highly conductive ambient-temperature molten salts. Inorg. Chem. 1996, 35, 1168-1178. [CrossRef] [PubMed]

6. Yu, B.; Zhou, F.; Mu, Z.; Liang, Y.; Liu, W. Tribological properties of ultra-thin ionic liquid films on single-crystal silicon wafers with functionalized surfaces. Tribol. Intern. 2006, 39, 879-887. [CrossRef] 
7. Somers, A.; Howlett, P.; MacFarlane, D.; Forsyth, M. A review of ionic liquid lubricants. Lubricants 2013, 1, 3-21. [CrossRef]

8. Mo, Y.; Yu, B.; Zhao, W.; Bai, M. Microtribological properties of molecularly thin carboxylic acid functionalized imidazolium ionic liquid film on single-crystal silicon. Appl. Surf. Sci. 2008, 255, 2276-2283.

9. Bhushan, B.; Palacio, M.; Kinzig, B. AFM-based nanotribological and electrical characterization of ultrathin wear-resistant ionic liquid films. J. Colloid Interf. Sci. 2008, 317, 275-287. [CrossRef] [PubMed]

10. Lertola, A.C.; Wang, B.; Li, L. Understanding the friction of nanometer-thick fluorinated ionic liquids. Ind. Eng. Chem. Res. 2018, 57, 11681-11685.

11. Zhao, W.; Pu, J.; Yu, Q.; Zeng, Z.; Wu, X.; Xue, Q. A novel strategy to enhance micro/nano-tribological properties of DLC film by combining micro-pattern and thin ionic liquids film. Colloids Surf. A Physicochem. Eng. Asp. 2013, 428, 70-78.

12. Zhao, W.; Wang, Y.; Wang, L.; Bai, M.; Xue, Q. Influence of heat treatment on the micro/nano-tribological properties of ultra-thin ionic liquid films on silicon. Colloids Surf. A Physicochem. Eng. Asp. 2010, 361, 118-125. [CrossRef]

13. Zhang, L.; Pu, J.; Wang, L.; Xue, Q. Frictional dependence of graphene and carbon nanotube in diamond-like carbon/ionic liquids hybrid films in vacuum. Carbon 2014, 80, 734-745. [CrossRef]

14. Pu, J.; Wan, S.; Zhao, W.; Mo, Y.; Zhang, X; Wang, L.; Xue, Q. Preparation and tribological study of functionalized graphene-IL nanocomposite ultrathin lubrication films on Si substrates. J. Phys. Chem. C 2011, 115, 13275-13284. [CrossRef]

15. Pu, J.; Huang, D.; Wang, L.; Xue, Q. Tribology study of dual-layer ultrathin ionic liquid films with bonded phase: influences of the self-assembled underlayer. Colloids Surf. A Physicochem. Eng. Asp. 2010, 372, 155-164. [CrossRef]

16. Zhao, W.; Zhu, M.; Mo, Y.; Bai, M. Effect of anion on micro/nano-tribological properties of ultra-thin imidazolium ionic liquid films on silicon wafer. Colloids Surf. A Physicochem. Eng. Asp. 2009, 332, 78-83. [CrossRef]

17. Lv, M.; Han, F.; Wang, Q.; Wang, T.; Liang, Y. The structure properties and tribological behavior of the ionic liquid-polyimide composite films under high-vacuum environment. High Perform. Polym. 2017, 29, 170-177. [CrossRef]

18. Rasheed, A.K.; Khalid, M.; Rashmi, W.; Gupta, T.C.S.M.; Chan, A. Graphene based nanofluids and nanolubricants-review of recent developments. Renew. Sustain. Energy Rev. 2016, 63, 346-362. [CrossRef]

19. Xu, Y.; Peng, Y.; Dearn, K.D.; Zheng, X.; Yao, L.; Hu, X. Synergistic lubricating behaviors of graphene and $\mathrm{MoS}_{2}$ dispersed in esterified bio-oil for steel/steel contact. Wear 2015, 342-343, 297-309. [CrossRef]

20. Zhang, W.; Cao, Y.; Tian, P.; Guo, F.; Tian, Y.; Zheng, W.; Ji, X.; Liu, J. Soluble exfoliated two-dimensional nanosheets as excellent aqueous lubricants. ACS Appl. Mater. Int. 2016, 8, 32440-32449. [CrossRef]

21. Wu, X.; Gong, K.; Zhao, G.; Lou, W.; Wang, X.; Liu, W. Surface modification of $\mathrm{MoS}_{2}$ nanosheets as effective lubricant additives for reducing friction and wear in poly- $\alpha$-olefin. Ind. Eng. Chem. Res. 2018, 57, 8105-8114. [CrossRef]

22. Zhao, W.; Zeng, Z.; Peng, S.; Wu, X.; Xue, Q.; Chen, J. Fabrication and investigation the microtribological behaviors of ionic liquid-graphene composite films. Tribol. T 2013, 56, 480-487. [CrossRef]

23. Palacio, M.; Bhushan, B. Ultrathin wear-resistant ionic liquid films for novel MEMS/NEMS applications. Adv. Mater. 2008, 20, 1194-1198. [CrossRef]

24. Wang, Y.; Cao, X.A.; Lang, H.; Zeng, X.; Chen, B.; Chen, R.; Peng, Y. Enhanced Tribological properties of composite films based on ionic liquids with $\mathrm{MoS}_{2}$ nanosheets as additives. N. J. Chem. 2018, 42, 4887-4892. [CrossRef]

25. Zhao, J.; He, Y.; Wang, Y.; Wang, W.; Yan, L.; Luo, J. An investigation on the tribological properties of multilayer graphene and $\mathrm{MoS}_{2}$ nanosheets as additives used in hydraulic applications. Tribol. Int. 2016, 97, 14-20. [CrossRef]

26. Splendiani, A.; Sun, L.; Zhang, Y.; Li, T.; Kim, J.; Chim, C.Y.; Galli, G.; Wang, F. Emerging photoluminescence in monolayer $\mathrm{MoS}_{2}$. Nano Lett. 2010, 10, 1271-1275. [CrossRef]

27. Stankovich, S.; Dikin, D.A.; Piner, R.D.; Kohlhaas, K.A.; Kleinhammes, A.; Jia, Y.; Wu, Y.; Nguyen, S.T.; Ruoff, R.S. Synthesis of graphene-based nanosheets via chemical reduction of exfoliated graphite oxide. Carbon 2007, 45, 1558-1565. [CrossRef] 
28. Liang, S.; Shen, Z.; Yi, M.; Liu, L.; Zhang, X.; Ma, S. In-situ exfoliated graphene for high-performance water-based lubricants. Carbon 2016, 96, 1181-1190. [CrossRef]

29. Berman, D.; Erdemir, A.; Sumant, A.V. Reduced wear and friction enabled by graphene layers on sliding steel surfaces in dry nitrogen. Carbon 2013, 59, 167-175. [CrossRef]

30. Chua, C.K.; Pumera, M. Covalent chemistry on graphene. Chem. Soc. Rev. 2013, 42, 3222-3233. [CrossRef]

31. Jiang, J.W. Graphene versus $\mathrm{MoS}_{2}$ : A short review. Front. Phys. 2015, 10, 287-302. [CrossRef]

(C) 2020 by the authors. Licensee MDPI, Basel, Switzerland. This article is an open access article distributed under the terms and conditions of the Creative Commons Attribution (CC BY) license (http:/ / creativecommons.org/licenses/by/4.0/). 